\title{
Relationship Between the Triglyceride Glucose Index and the Risk of First Stroke in Elderly Hypertensive Patients
}

\author{
Longlong Hu $\mathbb{D}^{1}$, Huihui Bao ${ }^{1,2}$, Xiao Huang $\mathbb{D}^{\prime}$, Wei Zhou ${ }^{1,2}$, Tao Wang ${ }^{1,2}$, Lingjuan Zhu ${ }^{1,2}$, Xi Liu ${ }^{3}$, \\ Minghui $\mathrm{Li}^{1,3}$, Xiaoshu Cheng ${ }^{1,2}$ \\ 'Department of Cardiovascular Medicine, The Second Affiliated Hospital of Nanchang University, Nanchang, Jiangxi, People's Republic of China; \\ ${ }^{2}$ Center for Prevention and Treatment of Cardiovascular Diseases, The Second Affiliated Hospital of Nanchang University, Nanchang, Jiangxi, People's \\ Republic of China; ${ }^{3}$ Center of Cardiovascular Medicine, Inner Mongolia People's Hospital, Hohhot, Inner Mongolia, People's Republic of China \\ Correspondence: Minghui Li, Center of Cardiovascular Medicine, Inner Mongolia People's Hospital, No. 20 Zhaowuda Road, Hohhot, Inner Mongolia, \\ 010017, People's Republic of China, Tel +8615389819973, Fax+86-791-86262262, Email 15389819973@I63.com; Xiaoshu Cheng, Department of \\ Cardiovascular Medicine, The Second Affiliated Hospital of Nanchang University, No. I Minde Road, Nanchang, Jiangxi, 330006, People's Republic of \\ China, Tel +86I3607089 I28, Fax+86-79I-86262262, Email xiaoshumenfan I26@|63.com
}

Background: Several recent studies have shown the relationship between the triglyceride glucose (TyG) index and the risk of stroke in the general population and in a few patient cohorts; however, the role of the TyG index on stroke risk in elderly hypertensive patients has not been determined. Thus, we aimed to investigate the association of the TyG index with first stroke and first ischemic stroke in elderly individuals with hypertension.

Methods: We included 8487 elderly subjects with hypertension from the China H-type Hypertension Registry Study for the current analysis. The TyG index was calculated as $\ln$ (fasting triglyceride $[\mathrm{mg} / \mathrm{dL}] \times$ fasting glucose $[\mathrm{mg} / \mathrm{dL}] / 2$ ). Outcomes were the first stroke and first ischemic stroke.

Results: During a median follow-up of 1.72 years, the first stroke was diagnosed in 82 patients $(0.97 \%)$, and the first ischemic stroke was diagnosed in 48 patients $(0.57 \%)$. Multivariable Cox proportional hazards models revealed that the TyG index was positively associated with the risk of first stroke (per 1-unit increment; HR: 1.72; 95\% CI: 1.07, 2.76) and first ischemic stroke (HR: 2.31; 95\% CI: 1.32, 4.05). When the TyG index was assessed as quartiles, significantly higher risks of first stroke (HR: 1.90; 95\% CI: 1.04, 3.45) and first ischemic stroke (HR: 2.45; 95\% CI: 1.16, 5.20) were found in participants in quartile 4 compared with those in quartiles 1-3. Conclusion: The TyG index is potentially useful in the early identification of elderly hypertensive patients at high risk of experiencing a first stroke.

Keywords: triglyceride glucose index, stroke, ischemic stroke, hypertension

\section{Introduction}

Stroke is a leading cause of morbidity and mortality worldwide ${ }^{1}$ and is of increasing concern in China. ${ }^{2}$ A recent national epidemiologic survey indicated that there are 2.4 million new stroke cases annually and 1.1 million stroke-related deaths in China. ${ }^{3}$ Among the pathological subtypes of stroke, ischemic stroke is the most common subtype, accounting for approximately $77.8 \%$ of the prevalent strokes. ${ }^{4}$ Current studies have shown that high blood pressure is the leading risk factor for the global disease burden, in particular, the risk of stroke. ${ }^{5,6}$ Additionally, being older and ageing are also associated with an increased risk of stroke but also with a rising blood pressure and a higher likelihood of suffering from hypertension. ${ }^{7,8}$ It has been reported that $77 \%$ of elderly patients with hypertension experience stroke. ${ }^{9}$ Given the ageing trend in the next decades, along with the increase in hypertension prevalence, ${ }^{8}$ there is a need to identify the population at high risk of stroke in elderly hypertensive patients and to deliver effective secondary prevention.

Insulin resistance (IR), a precursor and mechanism of type 2 diabetes, which has a strong relationship with atherosclerosis, ${ }^{10}$ diabetes, ${ }^{11}$ chronic kidney disease, ${ }^{12}$ and cancer. ${ }^{13}$ Accumulating evidence has also suggested that IR 
may have an important impact on the risk of stroke. ${ }^{10,14,15}$ The triglyceride-glucose (TyG) index, which is calculated by fasting glucose and triglycerides, has been reported to be significantly correlated with the hyperinsulinaemic euglycaemic clamp test and to be a reliable surrogate marker of IR. ${ }^{16}$ Several recent studies have shown an association between the TyG index and stroke in the general population. ${ }^{17-22}$ However, to our knowledge, the role of TyG index has not been examined in such a high-risk hypertensive older adult population. Therefore, this study aimed to specifically investigate the association of the TyG index with the first stroke and first ischemic stroke among elderly individuals with hypertension in the Chinese community.

\section{Methods}

\section{Study Design and Participants}

Data for our analysis were from the China H-type Hypertension Registry Study (CHRS registration number: ChiCTR1800017274). The CHRS study is an observational, real-world, ongoing prospective cohort study evaluating the treatment, related risk factors and prognosis of patients with hypertension in Wuyuan, Jiangxi Province, China. The study protocol and informed consent were approved by the Ethics Committee of the Anhui Medical University Biomedical Institute. All participants signed the written informed consent form.

The study design and methods of data collection have been described in detail previously. ${ }^{23}$ Briefly, from March 2018 to August 2018, a total of 14,268 hypertensive patients in the community were enrolled in the CHRS study. At each visit site, face-to-face interviews via a structured questionnaire, anthropometric measurements, and collection of blood samples were completed at baseline. The follow-up investigation was conducted from 31 August 2018 to 31 March 2020, and 14,227 individuals remained in the cohort. We excluded 4324 participants who were younger than 60 years old. After the exclusion of 750 participants with a history of stroke, 459 patients using glucose-lowering drugs, 201 patients using lipoprotein-lowering drugs, and 6 participants without data on the TyG index, a total of 8487 elderly hypertensive patients were selected for the final analysis (Supplementary Figure S1).

\section{Data Collection and Definition}

The participants underwent the following examinations: a standardized questionnaire, anthropometric measurements, and blood sample collection. The trained medical professionals carried out structured questionnaires, which included age, sex, education, smoking status, alcohol consumption, physical activity, current medication from pill bottles, and previous medical diagnoses. Physical examination items included height, weight, waist circumference (WC), and blood pressure (BP). Body mass index (BMI) was calculated as weight $(\mathrm{kg}) /$ height $\left(\mathrm{m}^{2}\right)$. BP was measured using electronic sphygmomanometer (Omron; Dalian, China) with the subject in a seated position after resting for $10 \mathrm{~min}$, and the average of the three measurements was used. Blood samples of all participants were obtained after an overnight fast of at least 8 hours and stored in the clinical laboratory of the National Clinical Research Center for Kidney Disease, Guangzhou, China. Fasting plasma glucose was assessed by the hexokinase method; serum total cholesterol and serum triglycerides (TGs) were measured by the oxidase method; serum high-density lipoprotein (HDL) cholesterol and serum low-density lipoprotein (LDL) cholesterol were analysed by the direct method; serum uric acid (SUA) was measured by the enzymatic uricase method; serum homocysteine was determined enzymatically; and serum creatinine was analysed by the picric acid method. The blood specimens were measured on automatic clinical analyzer (Beckman Coulter, AU680). The estimated glomerular filtration rate (eGFR) was estimated with the CKD-EPI equation. ${ }^{24}$ The TyG index was calculated as $\ln$ (fasting triglyceride $[\mathrm{mg} / \mathrm{dL}] \times$ fasting glucose $[\mathrm{mg} / \mathrm{dL}] / 2){ }^{25}$

\section{Study Outcomes}

The outcomes of the study were newly diagnosed stroke, including ischemic stroke. All participants were invited to attend an in-person follow-up visit from 31 August 2018 to 31 March 2020. At each follow-up visit, trained staff used a standard form to query medical history. If patients were hospitalized or had visited an emergency department, trained staff used the forms to record medical information: including medical history, treatments, and diagnosis at discharge from the participants' medical records. Stroke was recorded from hospital discharge diagnoses (The International 
Classification of Diseases, ICD-10). The list covers codes included I63 (Ischemic stroke (I63), I60-I61 (Hemorrhagic stroke) and I64 (no type stroke)), confirmed by computed tomography (CT), magnetic resonance imaging (MRI), transcranial, or carotid Doppler sonography. In addition, source data for all suspected stroke cases, including photocopies of the participant's inpatient record, discharge summary, biochemical tests, and imaging reports were submitted to the Outcome Adjudication Committee for further verification. All members of the committee were unaware of the baseline risk factors for the study participants.

\section{Statistical Analysis}

Baseline characteristics are presented as mean values \pm standard deviation (SDs) or median (interquartile range) for continuous variables and proportions for categorical variables. Analysis of variance, Kruskal-Wallis test, Fisher's exact test, or chi-square test were used to compare baseline characteristics by TyG index quartiles, as appropriate. Cox proportional hazards models were used to examine the association of the TyG index with the risk of first stroke and first ischemic stroke in four sequential models. Model 1 was only adjusted for age. Model 2 was additionally adjusted for sex, BMI, WC, education, physical activity, duration of hypertension, current smoking, and current drinking. Model 3 was further adjusted for SBP, DBP, serum homocysteine, SUA, LDL-C, and eGFR. Model 4 was adjusted for model 3 plus diabetes mellitus, atrial fibrillation, coronary heart disease (CHD), antihypertensive drugs, and antiplatelet drugs, which are variables that are known as traditional or suspected risk factors for stroke. ${ }^{26}$ Adjusted hazard ratios (HRs) and $95 \%$ confidence intervals (CIs) were estimated in each regression model. To assess the dose-response relationship of the TyG index with stroke and ischemic stroke, we used a Cox regression model with restricted cubic splines for the TyG index. Effect modification by sex was evaluated via stratified analyses and tested via the interaction term approach. In addition, we also estimated the incidence rates of stroke for each TyG quartile over the total follow-up period. Time-to-event curves were estimated using the Kaplan-Meier method, and the Log rank test was also conducted.

All statistical analyses were conducted with R software (version 3.3.1; http://www.R-project.org) and Empower (version 2.17.8; www.empowerstats.com; X\&Y Solutions). All $P$ values were 2 -sided, and a $P$ value $<0.05$ was considered statistically significant.

\section{Results}

\section{Basic Characteristics}

The baseline characteristics of the subjects according to the TyG index quartiles are shown in Table 1. In the study, a total of 8487 elderly hypertensive patients were evaluated. The mean age was 68.77 years, and $52.76 \%$ were men. The mean of baseline TyG index was 8.81. The higher quartiles of the TyG index were more likely to contain patients who were younger; were female; have a higher BMI, WC, SBP, and DBP; have a longer duration of hypertension; were not current smokers or current drinkers; have a history of diabetes mellitus; have a higher level of FPG, TC, TG, LDL-C, and SUA; have a lower level of HDL-C; and were using antihypertensive drugs. $(P<0.001)$.

\section{Association Between the TyG Index and First Stroke}

During an average follow-up of 1.72 years, there were 82 incident cases of stroke $(0.97 \%)$, including 48 patients with ischemic stroke $(0.57 \%)$. Overall, there was a significant positive association between the baseline TyG index and the risk of first stroke (Figure 1A). Table 2 shows the association of the TyG index with first stroke using multivariate Cox proportional hazard models. In models $1-4$, a continuous TyG index was positively correlated with incident stroke. In the fully adjusted model (model 4), each 1-unit increase in the TyG index was associated with a $72 \%$ increase in the adjusted risk of first stroke (HR: 1.72; 95\% CI: 1.07, 2.76). Consistently, when the TyG index was assessed as quartiles, the adjusted HRs for participants in quartiles 2, 3, and 4 were 1.34 (95\% CI: $0.65,2.74), 1.32$ (95\% CI: $0.59,2.95)$, and 2.40 (95\% CI: 1.05, 5.46), respectively, when compared with those in quartile $1(P$-trend $<0.05)$. A significantly higher risk of first stroke (HR: $1.90 ; 95 \%$ CI: 1.04 , 3.45 ) was found in participants in quartiles 4 compared with participants in quartile 1-3. 
Table I Baseline Characteristics of the Study Population According to TyG Index Quartiles

\begin{tabular}{|c|c|c|c|c|c|}
\hline & QI & Q2 & Q3 & Q4 & $P$-value \\
\hline $\mathrm{N}$ & 2122 & 2121 & 2122 & 2122 & \\
\hline Age (years) & $70.18 \pm 6.64$ & $69.24 \pm 6.34$ & $68.41 \pm 5.88$ & $67.26 \pm 5.46$ & $<0.001$ \\
\hline Male, n (\%) & $1427(67.25)$ & $1043(49.17)$ & $831(39.16)$ & 707 (33.32) & $<0.001$ \\
\hline Education, n (\%) & & & & & 0.356 \\
\hline$<$ high school & $1644(94.65)$ & $1609(94.31)$ & I586 (93.29) & $1612(93.78)$ & \\
\hline$\geq$ high school & $93(5.35)$ & $97(5.69)$ & $114(6.7 I)$ & $107(6.22)$ & \\
\hline BMI $\left(\mathrm{kg} / \mathrm{m}^{2}\right)$ & $21.22 \pm 3.07$ & $22.37 \pm 3.30$ & $23.50 \pm 3.27$ & $24.45 \pm 3.16$ & $<0.001$ \\
\hline$W C(\mathrm{~cm})$ & $77.47 \pm 8.79$ & $80.81 \pm 9.31$ & $84.32 \pm 9.45$ & $86.82 \pm 8.70$ & $<0.001$ \\
\hline Physical activity, n (\%) & & & & & 0.146 \\
\hline Mild & $990(56.99)$ & $988(57.91)$ & $1008(59.29)$ & $1048(60.97)$ & \\
\hline Moderate & $373(21.47)$ & $375(21.98)$ & $347(20.4 I)$ & $363(21.12)$ & \\
\hline Vigorous & $374(21.53)$ & $343(20.11)$ & 345 (20.29) & $308(17.92)$ & \\
\hline Current smoking, n (\%) & $824(38.83)$ & $614(28.95)$ & $497(23.43)$ & $44 I(20.79)$ & $<0.00$ I \\
\hline Current drinking, n (\%) & $660(31.10)$ & $480(22.63)$ & $412(19.42)$ & $374(17.63)$ & $<0.001$ \\
\hline $\mathrm{SBP}(\mathrm{mmHg})$ & $148.23 \pm 18.53$ & $149.81 \pm 17.75$ & $150.34 \pm 17.90$ & $150.78 \pm 18.64$ & $<0.001$ \\
\hline $\mathrm{DBP}(\mathrm{mmHg})$ & $85.80 \pm 10.79$ & $86.66 \pm 10.42$ & $86.88 \pm 10.16$ & $87.97 \pm 10.22$ & $<0.001$ \\
\hline FPG (mmol/L) & $5.43 \pm 0.60$ & $5.76 \pm 0.68$ & $6.00 \pm 0.87$ & $6.86 \pm 2.05$ & $<0.001$ \\
\hline $\mathrm{TC}(\mathrm{mmol} / \mathrm{L})$ & $4.65 \pm 0.92$ & $5.08 \pm 0.93$ & $5.38 \pm 1.04$ & $5.61 \pm 1.19$ & $<0.001$ \\
\hline TG (mmol/L) & $0.79 \pm 0.16$ & $1.18 \pm 0.17$ & $1.65 \pm 0.27$ & $2.95 \pm 1.32$ & $<0.00$ I \\
\hline Serum homocysteine, $\mu \mathrm{mol} / \mathrm{L}$ & $15.62(\mid 2.99-20.20)$ & $15.94(13.02-20.46)$ & $15.40(12.80-19.33)$ & $15.55(13.01-19.36)$ & $<0.001$ \\
\hline HDL-C (mmol/L) & $1.76 \pm 0.47$ & $1.68 \pm 0.42$ & $1.58 \pm 0.40$ & $1.44 \pm 0.38$ & $<0.001$ \\
\hline LDL-C (mmol/L) & $2.49 \pm 0.63$ & $2.86 \pm 0.67$ & $3.17 \pm 0.75$ & $3.34 \pm 0.84$ & $<0.001$ \\
\hline SUA, $\mu \mathrm{mol} / \mathrm{L}$ & $404.74 \pm 113.68$ & $406.57 \pm 116.33$ & $419.78 \pm 116.50$ & $449.44 \pm 126.14$ & $<0.001$ \\
\hline eGFR $\left(\mathrm{mL} / \mathrm{min}\right.$ per $\left.1.73 \mathrm{~m}^{2}\right)$ & $84.03 \pm 19.20$ & $83.96 \pm 19.08$ & $84.44 \pm 18.54$ & $83.20 \pm 19.61$ & 0.197 \\
\hline TyG index & $8.11 \pm 0.22$ & $8.58 \pm 0.11$ & $8.95 \pm 0.12$ & $9.59 \pm 0.37$ & $<0.001$ \\
\hline Duration of hypertension, (years) & $5.00(2.00-10.00)$ & $5.00(2.00-10.00)$ & $6.00(3.00-10.00)$ & $6.00(3.00-11.00)$ & $<0.001$ \\
\hline Diabetes mellitus, n (\%) & $38(1.79)$ & $58(2.73)$ & $119(5.61)$ & $278(13.10)$ & $<0.001$ \\
\hline Atrial fibrillation, $\mathrm{n}(\%)$ & $25(1.44)$ & $27(1.58)$ & $32(1.88)$ & $30(1.75)$ & 0.760 \\
\hline $\mathrm{CHD}, \mathrm{n}(\%)$ & $\mathrm{II} 7(5.5 \mathrm{I})$ & $108(5.09)$ & III (5.23) & $119(5.61)$ & 0.867 \\
\hline First stroke, n (\%) & $17(0.80)$ & $21(0.99)$ & $16(0.75)$ & $28(1.32)$ & 0.223 \\
\hline Antihypertensive drugs, n (\%) & $|3| \mid(6 \mid .78)$ & 1353 (63.79) & $1373(64.73)$ & 1475 (69.54) & $<0.00$ I \\
\hline Antiplatelet drugs, n (\%) & $35(1.65)$ & $34(1.60)$ & $41(1.93)$ & $38(1.79)$ & 0.844 \\
\hline
\end{tabular}

Note: Data are the mean \pm SD, median (interquartile range), or number (percentage).

Abbreviations: BMI, body mass index; WC, waist circumference; SBP, systolic blood pressure; DBP, diastolic blood pressure; FPG, fasting plasma glucose; TC, total cholesterol; TG, triglyceride; HDL-C, high-density lipoprotein cholesterol; LDL-C, low-density lipoprotein cholesterol; eGFR, estimated glomerular filtration rate; TyG, triglyceride glucose; CHD, coronary heart disease.

The association between the TyG index and the first ischemic stroke is presented in Figure 1B. Similarly, each 1-unit increase in the TyG index was associated with a 2.31-fold increase in the adjusted risk of first ischemic stroke (HR: 2.31; 95\% CI: 1.32, 4.05). Accordingly, participants in quartile 4 had a higher risk of first ischemic stroke (HR: $2.45 ; 95 \%$ CI: 1.16, 5.20) compared with participants in quartile 1-3 (Table 3).

The Kaplan-Meier survival curve for first stroke and ischemic stroke stratified by the TyG index is shown in Figure 2 . Compared with the quartile 1-3 of the TyG index, patients in quartile 4 had a higher risk of first stroke and ischemic stroke (log-rank $P<0.05)$.

\section{Sensitivity Analysis}

We further performed Cox regression analyses to assess the association of the TyG index and stroke after excluding patients with a history of diabetes or diabetes with obesity. Quartile 4 of the baseline TyG index was similarly associated with an increased risk of first stroke and first ischemic stroke in nondiabetic patients and nondiabetic obese patients 

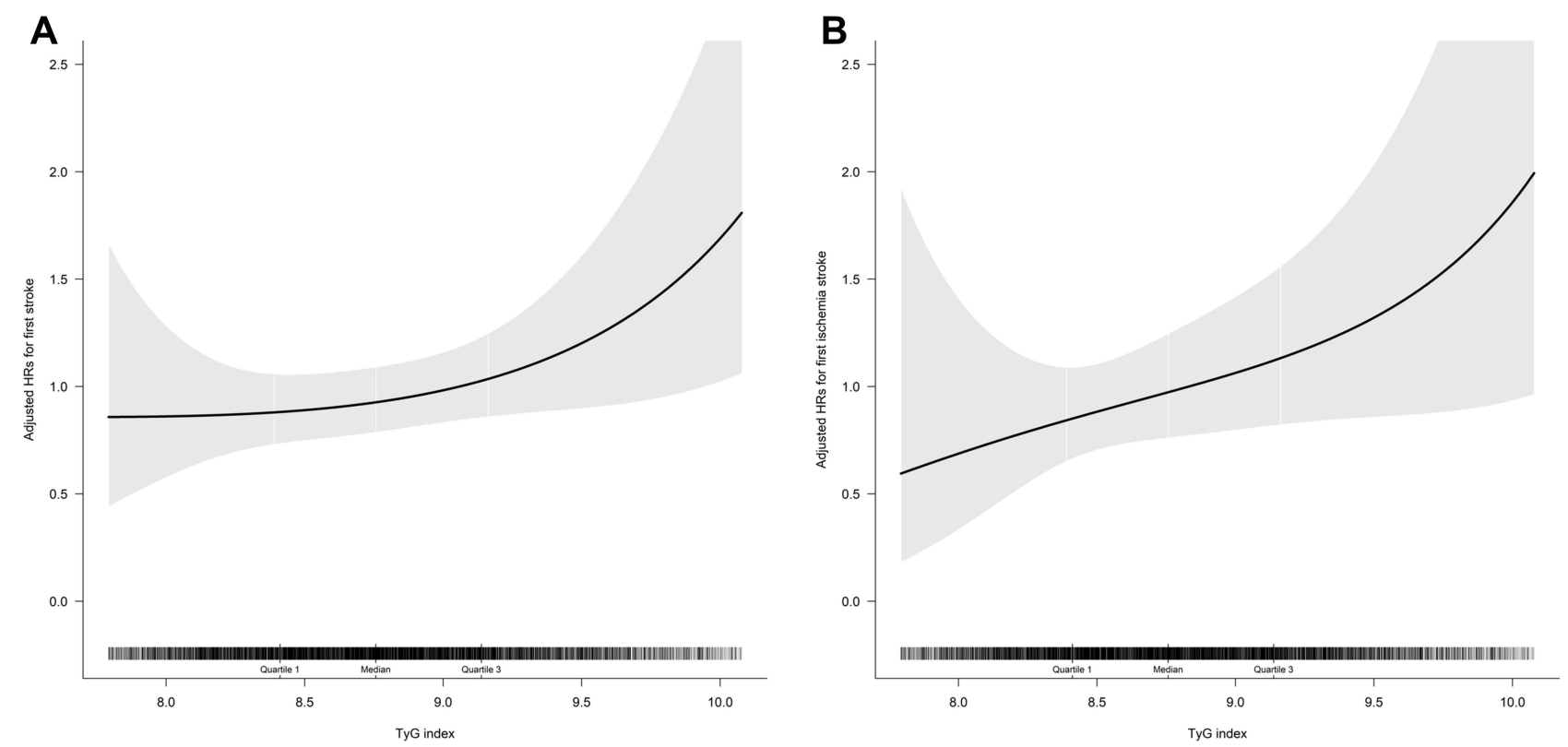

Figure I Dose-response associations of the TyG index with the risk of first stroke and first ischemic stroke*. (A) TyG index and first stroke; (B) TyG index and first ischemic stroke. *Adjusted for age, sex, BMI, WC, education, physical activity, duration of hypertension, current smoking, current drinking, BP, DBP, serum homocysteine, SUA, LDL-C, eGFR, diabetes mellitus, atrial fibrillation, CHD, anti-hypertensive drugs, anti-platelet drugs.

Abbreviation: TyG, triglyceride glucose.

(Supplementary Table S1). In addition, the association of TyG index with the first stroke and ischemic stroke were consistent in the different genders $(P$-interaction $=0.289$ and 0.768 , Supplementary Table S2 and Supplementary Figure S2).

\section{Discussion}

In this large-scale, prospective, hypertensive population-based cohort study, we found that higher levels of the TyG index at baseline were associated with an increased risk of first stroke and first ischemic stroke in elderly hypertensive patients. This finding persisted even after adjusting for as many cardiovascular risk factors as possible. This study contributes new

Table 2 The Association Between Baseline TyG Index and Incident Risk of First Stroke

\begin{tabular}{|c|c|c|c|c|c|}
\hline \multirow[t]{2}{*}{ TyG Index } & \multirow[t]{2}{*}{ Events (\%) } & \multicolumn{4}{|c|}{ First Stroke, HR (95\% Cl) } \\
\hline & & Model I & Model 2 & Model 3 & Model 4 \\
\hline Per I unit increase & $82 / 8487(0.97)$ & $1.53(1.07,2.20)$ & $1.82(1.19,2.80)$ & $1.72(1.07,2.75)$ & $1.72(1.07,2.76)$ \\
\hline \multicolumn{6}{|l|}{ Quartiles } \\
\hline Q I (7.15-8.38) & $17 / 2122(0.80)$ & I & I & I & $\mathrm{I}$ \\
\hline Q 2 (8.39-8.75) & $2 \mathrm{I} / 2 \mathrm{I} 2 \mathrm{I}(0.99)$ & $1.32(0.69,2.50)$ & $1.45(0.72,2.95)$ & $1.30(0.63,2.65)$ & $1.34(0.65,2.74)$ \\
\hline Q 3 (8.76-9.15) & $16 / 2122(0.75)$ & $1.07(0.54,2.12)$ & $1.42(0.66,3.09)$ & $1.31(0.58,2.93)$ & $1.32(0.59,2.95)$ \\
\hline Q $4(9.16-11.73)$ & $28 / 2122(1.32)$ & $2.04(1.11,3.78)$ & $2.67(1.26,5.64)$ & $2.34(1.04,5.28)$ & $2.40(1.05,5.46)$ \\
\hline$P$-trend & & 0.041 & 0.014 & 0.048 & 0.048 \\
\hline \multicolumn{6}{|l|}{ Categories } \\
\hline QI-Q3 (7.15-9.15) & $54 / 6365(0.85)$ & I & I & I & I \\
\hline Q4 (> 9.15) & $28 / 2122(1.32)$ & $1.81(1.14,2.88)$ & $2.04(1.17,3.56)$ & $1.88(1.04,3.40)$ & $1.90(1.04,3.45)$ \\
\hline
\end{tabular}

Notes: Data are regression coefficients ( $95 \%$ confidence interval). A test for linear trend was conducted with the use of quartiles of the TyG index as a continuous variable by assigning the median values of the quartiles to the variable. Model I is adjusted for age. Model 2 is further adjusted for sex, BMI, WC, education, physical activity, duration of hypertension, current smoking, current drinking. Model 3 is further adjusted for SBP, DBP, serum homocysteine, SUA, LDL-C, eGFR. Model 4 is further adjusted for diabetes mellitus, atrial fibrillation, CHD, anti-hypertensive drugs, anti-platelet drugs.

Abbreviations: TyG, triglyceride glucose; HR, hazard ratio; $\mathrm{Cl}$, confidence interval. 
Table 3 The Association Between Baseline TyG Index and Incident Risk of First Ischemic Stroke

\begin{tabular}{|c|c|c|c|c|c|}
\hline \multirow[t]{2}{*}{ TyG Index } & \multirow[t]{2}{*}{ Events (\%) } & \multicolumn{4}{|c|}{ First Ischemic Stroke, HR (95\% Cl) } \\
\hline & & Model I & Model 2 & Model 3 & Model 4 \\
\hline Per I unit increase & & $2.23(1.43,3.48)$ & $2.47(1.47,4.13)$ & $2.31(1.32,4.05)$ & $2.31(1.32,4.05)$ \\
\hline Quartiles & $48 / 8487(0.57)$ & & & & \\
\hline Q I (7.15-8.38) & $6 / 2122(0.28)$ & I & I & 1 & I \\
\hline Q 2 (8.39-8.75) & $|3 / 2| 2 \mid(0.61)$ & $2.32(0.88,6.10)$ & $2.33(0.85,6.42)$ & $2.09(0.75,5.79)$ & $2.14(0.77,5.95)$ \\
\hline Q 3 (8.76-9.15) & $9 / 2122(0.42)$ & I.7I $(0.60,4.82)$ & $1.72(0.55,5.38)$ & $1.49(0.46,4.85)$ & $1.52(0.47,4.94)$ \\
\hline Q 4 (9.16-11.73) & $20 / 2122(0.94)$ & $4.15(1.65,10.49)$ & $4.63(1.62,13.26)$ & $3.92(1.26,12.16)$ & $3.99(1.28,12.48)$ \\
\hline$P$-trend & & 0.004 & 0.007 & 0.028 & 0.028 \\
\hline \multicolumn{6}{|l|}{ Categories } \\
\hline QI-Q3 (7.15-9.15) & $28 / 6365(0.44)$ & I & $\mathrm{I}$ & I & I \\
\hline Q4 (> 9.15) & $20 / 2122(0.94)$ & $2.49(1.39,4.47)$ & $2.71(1.34,5.46)$ & $2.46(1.17,5.18)$ & $2.45(1.16,5.20)$ \\
\hline
\end{tabular}

Notes: Data are regression coefficients ( $95 \%$ confidence interval). A test for linear trend was conducted with the use of quartiles of the TyG index as a continuous variable by assigning the median values of the quartiles to the variable. Model I is adjusted for age. Model 2 is further adjusted for sex, BMI, WC, education, physical activity, duration of hypertension, current smoking, current drinking. Model 3 is further adjusted for SBP, DBP, serum homocysteine, SUA, LDL-C, eGFR. Model 4 is further adjusted for diabetes mellitus, atrial fibrillation, CHD, anti-hypertensive drugs, anti-platelet drugs.

Abbreviations: TyG, triglyceride glucose; $\mathrm{HR}$, hazard ratio; $\mathrm{Cl}$, confidence interval.

information concerning older adults with hypertension, a group in whom IR is common and stroke risk is high, and for whom data are relatively lacking from prior studies.

Recently, several studies have shown an association between the TyG index and stroke. In a cross-sectional study of 10,900 subjects from China, ${ }^{20} \mathrm{Shi}$ et al found the TyG index was positively associated with ischemic stroke. Data from the Kailuan Study, ${ }^{19}$ a study of 97,653 participants free of a history of stroke during a median of 11.02 years of follow-up by Wang et al, found that both the baseline and long-term updated cumulative average TyG index can predict stroke and ischemic stroke but not intracerebral haemorrhage. Zhao et $\mathrm{al}^{22}$ and Hong et $\mathrm{al}^{17}$ consistently reported that a high TyG index was significantly associated with an increased risk of stroke. In addition, a Mendelian randomization study by Si et al ${ }^{18}$ of 273,368 individuals of European ancestry showed that the TyG index was significantly associated with several cerebrovascular diseases. Collectively, these findings suggested that the TyG index, a simple measure reflecting insulin resistance, was strongly correlated with the risk of stroke. However, apart from a few patient cohort studies, ${ }^{27-29}$ most of the evidence is derived from general populations, rather than from stroke event-driven studies in high-risk individuals. Given older adults with hypertension represent a specific high-risk group for accelerated incident stroke, ${ }^{30}$ whether the TyG index predicts the

A $\quad$ TyG index $+01-03+04$

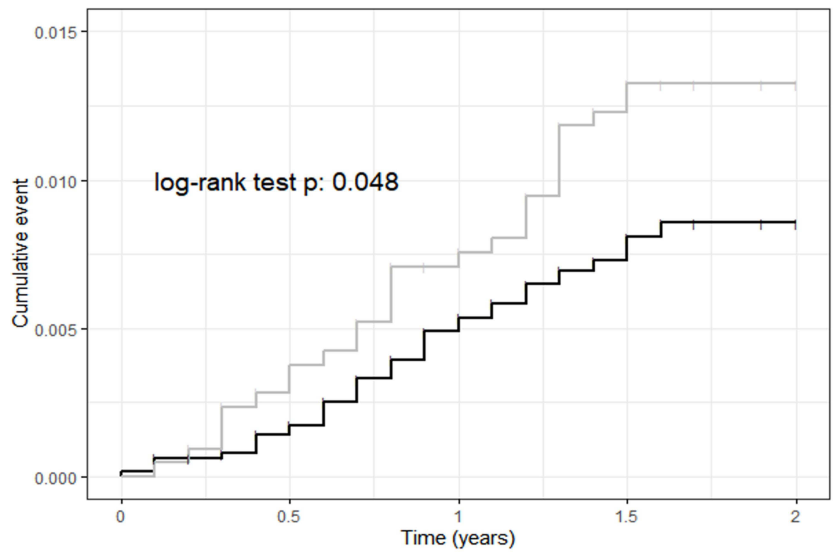

B TyG index $+Q 1-03+04$

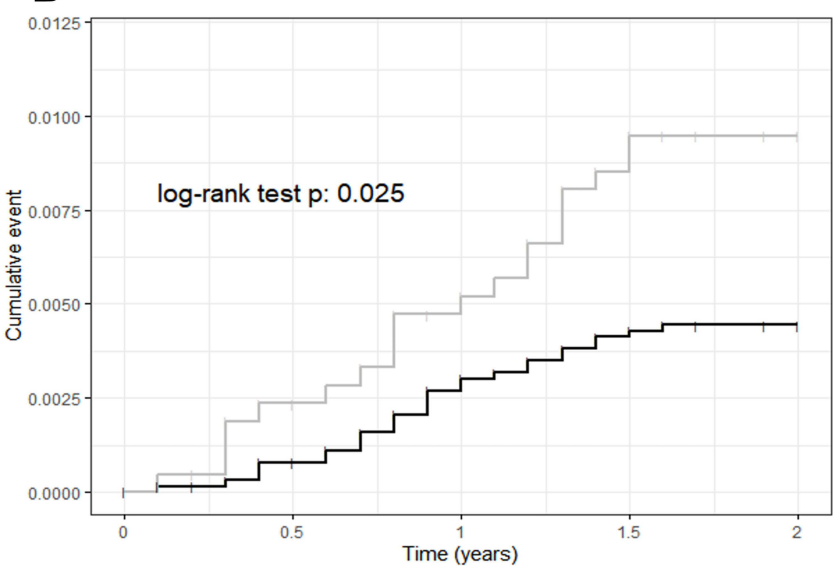

Figure 2 Kaplan-Meier curves of incidence of first stroke and first ischemic stroke according to the baseline TyG index. (A) TyG index and first stroke; (B) TyG index and first ischemic stroke.

Abbreviation: TyG, triglyceride glucose. 
first stroke outcome remains uncertain. Therefore, we conducted our study to investigate the association. Consistent with previous studies, the results in the main analysis of the study support that a higher TyG index was significantly associated with an increased risk of future stroke in elderly patients with hypertension.

The exact mechanism underlying the relationship between the TyG index and stroke has not been fully elucidated. The TyG index has been proposed as a reliable marker of IR. ${ }^{25,31}$ Thus, IR may mainly account for these associations. First, IR plays an important role in endothelial dysfunction, ${ }^{32}$ chronic inflammation, ${ }^{33}$ and platelet adhesion, activation, and aggregation. ${ }^{34,35}$ Second, IR was associated with the development of atherosclerosis, plaque progression, and rupture. ${ }^{29,36,37}$ Third, IR may be a mechanism that leads to hypertension, diabetes, ${ }^{11}$ dyslipidaemia, and other factors, ${ }^{38}$ ultimately increasing stroke risk. ${ }^{14}$ Additionally, the TyG index, which is calculated from fasting glucose and triglycerides, was positively associated with hyperglycaemia and dyslipidaemia, suggesting that the TyG index may influence stroke risk through the presence of cardiovascular risk factors.

There are some limitations in this study. First, glycosylated haemoglobin and 2-h oral glucose tolerance tests were not examined, so the impact of impaired glucose homeostasis on stroke could not be assessed. Second, although the most available demographic and clinical variables were included in the regression models, residual confounding factors could have affected the results. Third, due to the sample size being too small for haemorrhagic stroke, we did not analyse this type of stroke separately. Fourth, the TyG index was only assessed at baseline, and more frequent measurements would possibly have provided more information. Finally, the study was limited to a specific subpopulation, so our findings cannot be extrapolated to other populations.

In summary, using data from a prospective, large-scale hypertension registry study, we found that a high TyG index representing IR was independently associated with an increased risk of first stroke. These findings suggested that the TyG index may be a useful marker for risk stratification in elderly patients with hypertension.

\section{Data Sharing Statement}

The datasets used and/or analyzed in the current study are available from the corresponding author upon reasonable request.

\section{Ethics Approval and Consent to Participate}

The study was conducted in accordance with the Declaration of Helsinki and was approved by the Institute of Biomedicine, Anhui Medical University, Hefei, China. Informed written consent was obtained from all patients before their enrollment in this study.

\section{Acknowledgments}

Thanks to the investigators and subjects who participated in the China Hypertension Registry Study.

\section{Author Contributions}

All authors made substantial contributions to conception and design, acquisition of data, or analysis and interpretation of data; took part in drafting the article or revising it critically for important intellectual content; agreed to submit to the current journal; gave final approval of the version to be published; and agree to be accountable for all aspects of the work.

\section{Funding}

This work was supported by the Science and Technology Innovation Platform Project of Jiangxi Province (Grant number: 20165BCD41005).

\section{Disclosure}

The authors report no conflicts of interest in this work. 


\section{References}

1. Krishnamurthi RV, Ikeda T, Feigin VL. Global, regional and country-specific burden of ischaemic stroke, intracerebral haemorrhage and subarachnoid haemorrhage: a systematic analysis of the Global Burden of Disease Study 2017. Neuroepidemiology. 2020;54(2):171-179. doi: $10.1159 / 000506396$

2. Zhou M, Wang H, Zeng X, et al. Mortality, morbidity, and risk factors in China and its provinces, 1990-2017: a systematic analysis for the Global Burden of Disease Study 2017. Lancet. 2019;394(10204):1145-1158. doi:10.1016/S0140-6736(19)30427-1

3. Wu S, Wu B, Liu M, et al. Stroke in China: advances and challenges in epidemiology, prevention, and management. Lancet Neurol. 2019;18 (4):394-405. doi:10.1016/S1474-4422(18)30500-3

4. Han L, Liu Y, Wang C, et al. Determinants of hyperhomocysteinemia in healthy and hypertensive subjects: a population-based study and systematic review. Clin Nutr. 2017;36(5):1215-1230. doi:10.1016/j.clnu.2016.11.011

5. Lim SS, Vos T, Flaxman AD, et al. A comparative risk assessment of burden of disease and injury attributable to 67 risk factors and risk factor clusters in 21 regions, 1990-2010: a systematic analysis for the Global Burden of Disease Study 2010. Lancet. 2012;380(9859):2224-2260.

6. Thompson AM, Hu T, Eshelbrenner CL, Reynolds K, He J, Bazzano LA. Antihypertensive treatment and secondary prevention of cardiovascular disease events among persons without hypertension: a meta-analysis. JAMA. 2011;305(9):913-922. doi:10.1001/jama.2011.250

7. Franklin SS, Wong ND. Hypertension and cardiovascular disease: contributions of the Framingham heart study. Glob Heart. 2013;8(1):49-57. doi:10.1016/j.gheart.2012.12.004

8. Lloyd-Jones DM, Evans JC, Levy D. Hypertension in adults across the age spectrum: current outcomes and control in the community. JAMA. 2005;294(4):466-472. doi:10.1001/jama.294.4.466

9. Aronow WS, Fleg JL, Pepine CJ, et al. ACCF/AHA 2011 expert consensus document on hypertension in the elderly: a report of the American College of Cardiology Foundation Task Force on Clinical Expert Consensus Documents developed in collaboration with the American Academy of Neurology, American Geriatrics Society, American Society for Preventive Cardiology, American Society of Hypertension, American Society of Nephrology, Association of Black Cardiologists, and European Society of Hypertension. J Am Soc Hypertens. 2011;5(4):259-352.

10. Ding X, Wang X, Wu J, Zhang M, Cui M. Triglyceride-glucose index and the incidence of atherosclerotic cardiovascular diseases: a meta-analysis of cohort studies. Cardiovasc Diabetol. 2021;20(1):76. doi:10.1186/s12933-021-01268-9

11. Xuan X, Hamaguchi M, Cao Q, et al. U-shaped association between the triglyceride-glucose index and the risk of incident diabetes in people with normal glycemic level: a population-base longitudinal cohort study. Clin Nutr. 2021;40(4):1555-1561. doi:10.1016/j.clnu.2021.02.037

12. Okamura T, Hashimoto Y, Hamaguchi M, Obora A, Kojima T, Fukui M. Triglyceride-glucose index is a predictor of incident chronic kidney disease: a population-based longitudinal study. Clin Exp Nephrol. 2019;23(7):948-955. doi:10.1007/s10157-019-01729-2

13. Okamura T, Hashimoto Y, Hamaguchi M, Obora A, Kojima T, Fukui M. Triglyceride-glucose index (TyG index) is a predictor of incident colorectal cancer: a population-based longitudinal study. BMC Endocr Disord. 2020;20(1):113. doi:10.1186/s12902-020-00581-w

14. Kernan WN, Inzucchi SE, Viscoli CM, Brass LM, Bravata DM, Horwitz RI. Insulin resistance and risk for stroke. Neurology. 2002;59(6):809-815. doi:10.1212/WNL.59.6.809

15. Gast KB, Smit JW, den Heijer M, et al. Abdominal adiposity largely explains associations between insulin resistance, hyperglycemia and subclinical atherosclerosis: the NEO study. Atherosclerosis. 2013;229(2):423-429. doi:10.1016/j.atherosclerosis.2013.05.021

16. Guerrero-Romero F, Simental-Mendia LE, Gonzalez-Ortiz M, et al. The product of triglycerides and glucose, a simple measure of insulin sensitivity. Comparison with the euglycemic-hyperinsulinemic clamp. J Clin Endocrinol Metab. 2010;95(7):3347-3351. doi:10.1210/jc.2010-0288

17. Hong S, Han K, Park CY. The triglyceride glucose index is a simple and low-cost marker associated with atherosclerotic cardiovascular disease: a population-based study. BMC Med. 2020;18(1):361. doi:10.1186/s12916-020-01824-2

18. Si S, Li J, Li Y, et al. Causal effect of the triglyceride-glucose index and the joint exposure of higher glucose and triglyceride with extensive cardio-cerebrovascular metabolic outcomes in the UK Biobank: a Mendelian Randomization Study. Front Cardiovasc Med. $2020 ; 7: 583473$. doi:10.3389/fcvm.2020.583473

19. Wang A, Wang G, Liu Q, et al. Triglyceride-glucose index and the risk of stroke and its subtypes in the general population: an 11-year follow-up. Cardiovasc Diabetol. 2021;20(1):46. doi:10.1186/s12933-021-01238-1

20. Shi W, Xing L, Jing L, et al. Value of triglyceride-glucose index for the estimation of ischemic stroke risk: insights from a general population. Nutr Metab Cardiovasc Dis. 2020;30(2):245-253.

21. Sanchez-Inigo L, Navarro-Gonzalez D, Fernandez-Montero A, Pastrana-Delgado J, Martinez JA. Risk of incident ischemic stroke according to the metabolic health and obesity states in the vascular-metabolic CUN cohort. Int J Stroke. 2017;12(2):187-191. doi:10.1177/1747493016672083

22. Zhao Y, Sun H, Zhang W, et al. Elevated triglyceride-glucose index predicts risk of incident ischaemic stroke: the Rural Chinese Cohort Study. Diabetes Metab. 2021;47(4):101246. doi:10.1016/j.diabet.2021.101246

23. Li M, Zhan A, Huang X, et al. Positive association between triglyceride glucose index and arterial stiffness in hypertensive patients: the China H-type Hypertension Registry Study. Cardiovasc Diabetol. 2020;19(1):139. doi:10.1186/s12933-020-01124-2

24. Levey AS, Stevens LA, Schmid CH, et al. A new equation to estimate glomerular filtration rate. Ann Intern Med. 2009;150(9):604-612. doi:10.7326/0003-4819-150-9-200905050-00006

25. Simental-Mendia LE, Rodriguez-Moran M, Guerrero-Romero F. The product of fasting glucose and triglycerides as surrogate for identifying insulin resistance in apparently healthy subjects. Metab Syndr Relat Disord. 2008;6(4):299-304. doi:10.1089/met.2008.0034

26. Meschia JF, Bushnell C, Boden-Albala B, et al. Guidelines for the primary prevention of stroke: a statement for healthcare professionals from the American Heart Association/American Stroke Association. Stroke. 2014;45(12):3754-3832.

27. Mao Q, Zhou D, Li Y, Wang Y, Xu SC, Zhao XH. The triglyceride-glucose index predicts coronary artery disease severity and cardiovascular outcomes in patients with non-ST-segment elevation acute coronary syndrome. Dis Markers. 2019;2019:6891537. doi:10.1155/2019/6891537

28. Wang L, Cong HL, Zhang JX, et al. Triglyceride-glucose index predicts adverse cardiovascular events in patients with diabetes and acute coronary syndrome. Cardiovasc Diabetol. 2020;19(1):80. doi:10.1186/s12933-020-01054-Z

29. Ma X, Dong L, Shao Q, et al. Triglyceride glucose index for predicting cardiovascular outcomes after percutaneous coronary intervention in patients with type 2 diabetes mellitus and acute coronary syndrome. Cardiovasc Diabetol. 2020;19(1):31. doi:10.1186/s12933-020-01006-7

30. Wang W, Jiang B, Sun H, et al. Prevalence, incidence, and mortality of stroke in China: results from a Nationwide Population-Based Survey of 480 687 adults. Circulation. 2017;135(8):759-771. doi:10.1161/CIRCULATIONAHA.116.025250 
31. Du T, Yuan G, Zhang M, Zhou X, Sun X, Yu X. Clinical usefulness of lipid ratios, visceral adiposity indicators, and the triglycerides and glucose index as risk markers of insulin resistance. Cardiovasc Diabetol. 2014;13:146. doi:10.1186/s12933-014-0146-3

32. Lteif AA, Han K, Mather KJ. Obesity, insulin resistance, and the metabolic syndrome: determinants of endothelial dysfunction in whites and blacks. Circulation. 2005;112(1):32-38. doi:10.1161/CIRCULATIONAHA.104.520130

33. Bloomgarden ZT. Inflammation and insulin resistance. Diabetes Care. 2003;26(5):1619-1623. doi:10.2337/diacare.26.5.1619

34. Kain K, Catto AJ, Grant PJ. Associations between insulin resistance and thrombotic risk factors in high-risk South Asian subjects. Diabet Med. 2003;20(8):651-655. doi:10.1046/j.1464-5491.2003.00958.x

35. Vinik AI, Erbas T, Park TS, Nolan R, Pittenger GL. Platelet dysfunction in type 2 diabetes. Diabetes Care. 2001;24(8):1476-1485.

36. Bornfeldt KE, Tabas I. Insulin resistance, hyperglycemia, and atherosclerosis. Cell Metab. 2011;14(5):575-585. doi:10.1016/j.cmet.2011.07.015

37. Alizargar J, Bai $\mathrm{CH}$. Comparison of carotid ultrasound indices and the triglyceride glucose index in hypertensive and normotensive community-dwelling individuals: a Case Control Study for evaluating atherosclerosis. Medicina (Kaunas). 2018;54(5):71.

38. Kitae A, Hashimoto Y, Hamaguchi M, Obora A, Kojima T, Fukui M. The Triglyceride and Glucose Index Is a predictor of incident nonalcoholic fatty liver disease: a Population-Based Cohort Study. Can J Gastroenterol Hepatol. 2019;2019:5121574.

\section{Publish your work in this journal}

The International Journal of General Medicine is an international, peer-reviewed open-access journal that focuses on general and internal medicine, pathogenesis, epidemiology, diagnosis, monitoring and treatment protocols. The journal is characterized by the rapid reporting of reviews, original research and clinical studies across all disease areas. The manuscript management system is completely online and includes a very quick and fair peer-review system, which is all easy to use. Visit http://www.dovepress.com/testimonials.php to read real quotes from published authors.

Submit your manuscript here: https://www.dovepress.com/international-journal-of-general-medicine-journal 